\title{
Early Diagnosis of Keratic Mycosis: A Call of Hours !!
}

\author{
Sarika Kombade*, Padma Das, Neeta Gade, Ujjwala Gaikwad, Sanjay S Negi and Anudita Bhargava \\ Department of Microbiology, AIIMS Jodhpur, India
}

*Corresponding Author: Sarika Kombade, Assistant Professor, Department of Microbiology, AIIMS Jodhpur, India.

Received: August 06, 2019; Published: October 17, 2019

DOI: $10.31080 /$ ASMI.2019.02.0404

\begin{abstract}
Mycotic keratitis is one of the leading causes of ocular morbidity and blindness in tropical countries like India [4] Lack of awareness, delayed diagnosis and inappropriate treatment contributes to increasing burden of the disease [4] Aspergillus spp. and Candida albicans stand out as the major offending fungal pathogens where as Acremonium spp. contributes only to $6.6 \%$ of the total mycotic keratitis cases in Indian scenario [5,6].

This case, A 27 year old male farmer presented to the ophthalmology outdoor department with photophobia, eye discharge, reduced vision and pain in eye with the history of corneal injury 8 days back. Patient was not having any systemic illness like diabetes, hypertension. Past history was not suggestive of any previous ocular infections. Patient had started earlier treatment with oral ciprofloxacin and topical itraconazole for 5 days. Sarika Kombade, Padma Das, Neeta Gade, Ujjwala Gaikwad, Sanjay S. Negi, Anudita Bhargava.
\end{abstract}

Keywords: Mycotic keratitis; Aspergillus spp; Candida albicans

\section{Introduction}

Incidence of corneal trauma and posttraumatic infections leading to monocular blindness are common occurrences in Indian scenario. Microbial corneal diseases are reported as major causes of morbidity and blindness worldwide accounting about ten million cases annually [1]. Outcome of fungal keratitis are unfavorable due to protracted course of the condition and diversity of clinical presentation. More than 105 species of fungi such as Aspergillus spp, Fusarium spp, Candida spp., Rhizopus, Mucor and other fungi have been identified as the etiological agents of fungal keratitis [2]. Genus Acremonium belong to ascomycetes group amounting up to 100 species. Most of the Acremonium spp. are environmental saprophytes but few species like A. falciforme, A. kiliense, A. recifei etc behave as human pathogens. There are worldwide reported cases of Acremonium spp. Infections presenting as onychomycosis, keratitis, endophthalmitis, endocarditis, meningitis and peritonitis. These are more common in immunocompromised patients such as different organ transplant recipients; HIV infected patients whereas presence of invasive Acremonial infections in immunocompetent persons cannot be ruled out in some rare cases [3].
Mycotic keratitis is one of the leading causes of ocular morbidity and blindness in tropical countries like India [4]. Lack of awareness, delayed diagnosis and inappropriate treatment contributes to increasing burden of the disease [4]. Aspergillus spp. and Candida albicans stand out as the major offending fungal pathogens where as Acremonium spp. contributes only to $6.6 \%$ of the total mycotic keratitis cases in Indian scenario $[5,6]$.

This case, A 27-year-old male farmer presented to the ophthalmology outdoor department with photophobia, eye discharge, reduced vision and pain in eye with the history of corneal injury 8 days back. Patient was not having any systemic illness like diabetes, hypertension. Past history was not suggestive of any previous ocular infections. Patient had started earlier treatment with oral ciprofloxacin and topical itraconazole for 5 days.

\section{Materials and Methods}

All the routine examinations were within normal range. The corneal scrapping sent to microbiology department showed presence of thin hyaline branching hyphae on $10 \% \mathrm{KOH}$ mount. Gram 
stained smear revealed partially stained gram positive hyphal structure. The culture on SDA after 5 days of incubation revealed a rapidly growing pinkish white rugose fungal colony with brown reverse diffusible pigment in the medium (Figure 1 and 2). The LPCB mount and microslide culture were done to observe exact morphology of the fungal isolate. LPCB of microslide culture revealed hyaline septate hyphae with crescentic nonstate conidia arranged in loose cluster in a criss-cross formation at the tip of long slender delicate phialides in a blunt tip of conidiophore. Macroconidia were absent (Figure 3).

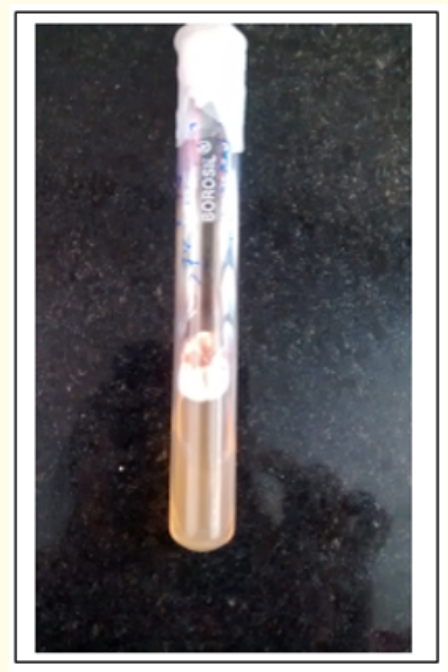

Figure 1: SDA showing obverse growth of colony.

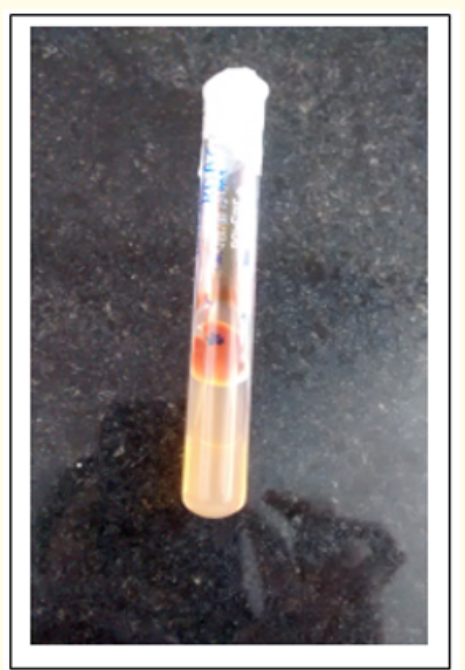

Figure 2: SDA showing pigmentation on reverse.

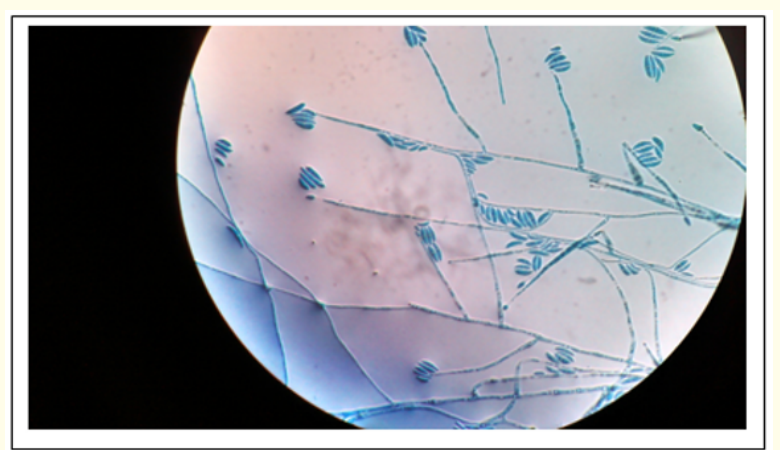

Figure 3: LPCB showing microconidia crescent shaped in loose cluster.

\section{Result and Discussion}

Acremonium spp. colonies are white, downy to wooly with pink to rose pigmentation. On microscopy Acremonium spp. shows conidia which are single or multicellular and fusiform with a slight curve, some appearing as crescents of size 10x4um in approximately. The different species vary in conidial structure [7]. A. falcifor$m e$ is mainly crescentic and nonseptate, but has some two-or three conidia. A. kiliense has straight short conidia; A. recifei is mainly crescentic and nonseptate [7].

Taking into account the appearance of the colony on Saboraud's Dextrose agar, rate of growth, reverse pigmentation and microscopic features the fungal isolate was identified to be Acremonium recifei as red diffusible pigment with microscopy having typical crescentic, nonseptate conidia arranged in loose cluster on conidiophore. After initial local surgical debridement, the patient responded well to oral Ketoconazole, topical Voriconazole and Natamycin therapy as observed in subsequent follow ups. Due to early diagnosis of fungal infection and identification of infecting fungus topical itraconazole therapy of the patient was changed to topical Voriconazole as the studies reported that antifungal therapy with fluconazole, amphotericin B, griseofulvin and itraconazole has been unsatisfactory in case of Acremonium keratitis with frequent relapses and progression to the posterior segment [3]. Patient responded to this topical antifungal very well.

Most Acremonium keratitis cases are underreported. As it is a rare cause of keratitis and considered as a common laboratory contaminant, but it can lead to exogenous endophthalmitis with very poor prognosis. In this case timely diagnosis, history of trauma to establish the fungal pathogenicity and early interventional measures accounted for the good recovery of the patient. 


\section{Conclusion}

To conclude, different fungal isolates have different antifungal sensitivity pattern. So it is important to identify the pathogenic fungus for appropriate treatment to reduce the incidence of complicated mycotic keratitis.

\section{Bibliography}

1. Whitcher JP., et al. "Corneal blindness: a global perspective". Bulletin of the World Health Organization 79.3 (2001): 214221.

2. Thomas PA. "Fungal infections of the cornea". Eye (Lond.) 17.8 (2003): 852-862.

3. Creti A., et al. "Voriconazole curative treatment for Acremonium species keratitis developed in a patient with concomitant Staphylococcus aureus corneal infection; a case report". In vivo 20 (2006): 162-172.

4. Siddiqui PA and Pandre S. "Infectious keratitis in present scenario". International Journal of Science and Research 2.10 (2013): 97-99.

5. Chander J and Sharma A. "Prevalence of fungal corneal ulcers in northen India”. Infection 22 (1994): 207-209.

6. Saha R and Das S. "Mycological profile of infectious keratitis from Delhi”. Indian Journal of Medical Research 123 (2006): 159-164.

7. Topley and Wilson`s Microbiology and microbial infections; medical mycology, 10th edition. William G. Merz and Rodrick J. Hay; Eumycetoma. Acremonium species". J phn Wiley and Sons-(2009): 390.

\section{Volume 2 Issue 11 November 2019}

(C) All rights are reserved by Sarika Kombade., et al. 